\title{
A Robust On-road Moving Platform Video Stabilization Using Derivative Curve Warping
}

\author{
Deepika Shukla ${ }^{1}$ and Rajib Kumar $\mathrm{Jha}^{2}$ \\ 1 PDPM Indian Institute of Information Technology, Design and Manufacturing \\ Jabalpur, India \\ 2 Indian Institute of Technology Patna, India
}

\begin{abstract}
In this paper a video stabilization technique based on derivative of integral projection curve has been proposed. The existing projection matching techniques consider only intensity values as the matching feature. Any change in pixel intensity values and their local distribution, results in shape variation of the projection curve. This shape variation affects the accuracy of curve matching and becomes a serious cause of wrong motion estimation. The proposed derivative curve technique considers the change in intensity values as the matching feature, instead of working on the intensity values itself. Inclusion of derivative provides shape information as high level feature and this results in better matching of two slightly dissimilar curves which are obvious for sequences taken from moving platform. Optimal matching of these derivative curves is achieved using Dynamic Time Warping technique.
\end{abstract}

Keywords: Integral projection, Dynamic time warping, Video stabilization.

\section{Introduction}

The videos captured using hand-held or moving platform camera are prone to undesired vibration induced motions. These undesired high frequency motions affect the visual quality of video sequence significantly. Video stabilization aims to remove the effect of such unwanted motions to provide a pleasant viewing experience. The relative motion between two consecutive frame is obtained using some motion estimation techniques. Accuracy of the estimated motion is highly desired as it directly reflects the performance of the whole stabilization system. In literature most of the motion estimation techniques are based on the block matching. The full block matching (BM) [1] technique provides accurate results in case pure translation but get affected by the presence of intensity variation or change in the local intensity distribution. It also suffers from large computational time. Robustness to intensity variation can be achieved using gray coded bit plane matching (GCBPM) instead of intensity frame and working on bit level reduces the processing time also 2. Hybrid of these techniques have also been proposed to improve the accuracy of the motion estimation by working on a portion of frame to reduce the computational complexity [3]. Reducing the 
matching area always results in a compromise in terms of accuracy in comparison to the full frame techniques. The use of integral projection (IP) 4] reduces the matching problem from the $2 \mathrm{D}$ frame to $1 \mathrm{D}$ intensity projection thus inheriting the full frame information with reduced processing time requirement. Accuracy of estimated motion depend on the accurate matching of these curves. Matching criterion of all the discussed techniques is based on correlation to get some error minimization which gets affected by the change in local intensity distribution. An efficient algorithm for the projection curve matching has been proposed using dynamic time warping (DTW) providing optimal alignment of the two curves with better accuracy [5]. The DTW algorithm provides better alignment in case the two curves are similar in shape but a small acceleration or deacceleration and difference in intensity levels of the curve produces singularity in the optimal warping path. Such variations in the projection curves are very obvious in the videos captured from moving platform, which mostly suffer from jerks induced displacement along with the change in frame content caused by forward zoom-in effect.

In this paper the use of derivative projection-curve is proposed for robust alignment of two curves. The use of derivative provides inclusion of high level feature 'shape', instead of working directly on the intensity values. Comparison of the DTW based Curve Warping (CW) technique [5] is done with the derivative curve warping (DCW) in case of moving platform video. Performance evaluation of whole stabilization system is done in terms of computed PSNR values over entire image sequence.

\section{Proposed Image Stabilization System}

The DTW finds alignment by selecting the intensity value of the projection curve and finds its best match in the target curve. This intensity based matching may cause one point to be matched with multiple points having same intensity, thus producing pathological results in curve alignment. The DTW algorithm fails to find obvious natural alignment in case the two curves having peak or valley difference (i.e. intensity value) and shape change caused by small acceleration (i.e. expansion) or deacceleration (i.e. contraction). This alignment problem could be overcome by using a higher level feature 'shape' instead of using the intensity values as the matching feature. This shape information is obtained using first derivative of the projection curve. Derivative curve focuses on how the curve is changing instead of instantaneous curve point values [6].

\subsection{Derivative Projection-Curve Extraction}

The intensity projection curves for a frame of size $R \times C$ in the horizontal and vertical directions are obtained using (1).

$$
\begin{aligned}
P_{H}(j) & =\frac{1}{R} \sum_{i=1}^{R} I(i, j) ; \forall j \in[1: C] \\
P_{V}(i) & =\frac{1}{C} \sum_{j=1}^{C} I(i, j) ; \forall i \in[1: R]
\end{aligned}
$$


where $I(i, j)$ represents the pixel luminance value at location $(i, j)$. Derivative curve $(D C)$ of the estimated intensity projection can be obtained using left point estimation. $D C$ along horizontal direction is given by (2).

$$
D C_{H}(j)=P_{H}(i)-P_{H}(i-1) ; \quad \text { where } \quad 1<i \leq C \quad \text { and } \quad j=i-1
$$

The obtained derivative curves of two consecutive frames are warped to find the optimal alignment of the respective intensity curves differing in their shape significantly.

\subsection{Motion Estimation}

Relative translational shift between two frames is clearly reflected in their respective projection curves. Adding the robustness in terms of variation in curve shape, instead of applying distance measure on the intensity curve, the difference of their derivative is proposed for better alignment. The distance matrix $D$ representing the squared difference of derivative of the two horizontal curves $D C_{H}$ of frame $f$ and $f-1$ is given by $(3)$.

$$
D_{f-1}^{f}(i, j)=\left(D C_{H}^{f}(i)-D C_{H}^{f-1}(j)\right)^{2}
$$

where $\forall i, j \in[1: C-1]$ and in case of $D C_{V}$ signature $\forall i, j \in[1: R-1]$. To reduced the processing time and memory requirement, warping of the derivative curves is done using dynamic programming. in this algorithm an accumulated distance matrix $A D$ is computed using recursive addition whose elements are given by (4).

$$
\begin{array}{cc}
A D(i, 1)=\sum_{k=1}^{i} D(k, 1) ; & \forall i \in[1: C-1] \\
A D(1, j)=\sum_{k=1}^{j} D(1, k) ; & \forall j \in[1: C-1] \\
A D(i, j)=\min [A D(i-1, j-1), A D(i-1, j), & A D(i, j-1)]+D(i, j) ; \\
& \forall i, j \in[2: C-1]
\end{array}
$$

In $A D$ matrix, the optimal warping path $P_{\text {opt }}$ corresponds to best curve alignment and is extracted by backtracking the minimum valued element in its three adjacent neighbors. The optimal path $P_{\text {opt }}$ starts with $P_{o p t}^{L}=(C-1, C-1)$ and ends with $P_{o p t}^{1}=(1,1)$. Other elements of optimal path $P_{o p t}^{l}$ can be obtained using (5):

$$
P_{o p t}^{l}:= \begin{cases}(1, j-1) ; & \text { if } i=1, \\ (i-1,1) ; & \text { if } j=1, \\ \operatorname{argmin}\{A D(i-1, j-1), & \\ A D(i-1, j), A D(i, j-1)\} ; & \text { otherwise. }\end{cases}
$$

The $P_{\text {opt }}$ obtained using proposed method provides best alignment and the uniqueness of path is ensured by following the lexicographical order in case the three neighbors have same value. To test the accuracy of proposed motion estimation algorithm, video ' $C A R$ ' is chosen. The sequence works as a representative 


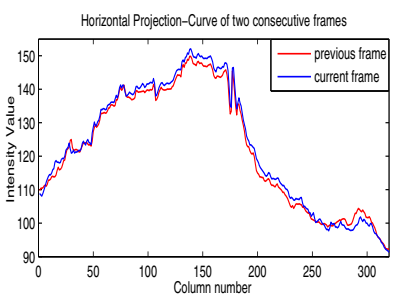

(a)

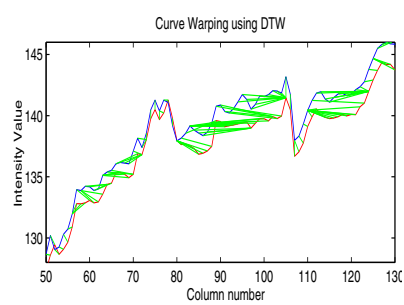

(b)

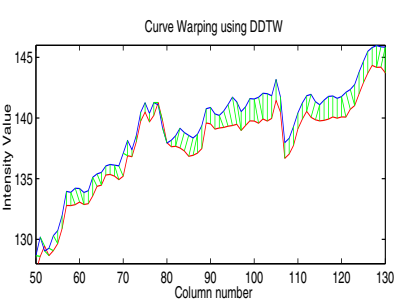

(c)

Fig. 1. (a) Shifted projection curves; Alignment using (b) CW (c) Proposed method

of real world moving platform videos. Figure 1(a) shows relative shift between two horizontal curves. To depict the effect of shape and intensity variation on accurate matching, the curve alignment using DTW based CW method and derivative DTW (DDTW) [6] based proposed DCW approach has been shown in Fig. 1(b) and Fig. 1(c) respectively. A portion of the two horizontal curves as shown in Fig. 1(a) has been selected for better visualization. Inclusion of shape information in the DDTW provides better alignment in comparison to the DTW, which suffers from the matching singularity (i.e. one to many point matching) at various location of curves.

\subsection{Motion Correction and Compensation}

To get the motion estimation with respect to a reference frame, an absolute GMV $(A G M V)$ for the each direction can be calculated using (6).

$$
A G M V^{f}=A G M V^{f-1}+G M V^{f}
$$

This $A G M V$ is used to separate the intentional panning from the jittery motion. Jitter being a high frequency oscillatory motion causes $A G M V$ to switch around certain value, while the intentional motion (panning) causes continuous shift in a single direction thus giving large motion vector values. Based on this concept a thresholding on the accumulated motion $A G M V$ can be used to separate out the two motions [1. The reference frame is changed in case the AGMV crosses the preset motion threshold and future motion estimation is done w.r.t. new reference frame. The negative of these calculated $A G M V$ s are used to compensate the effect of jitter by shifting the respective frame in opposite to the estimated direction.

\section{Experimental Results}

The moving platform videos suffer from the presence of large displacements caused due to platform jerks, and the forward motion of platform causes variation in the local pixel intensity distribution because of zoom-in effect. Existing 


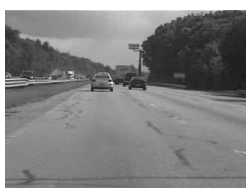

(a)

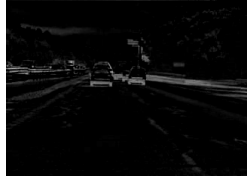

(b)

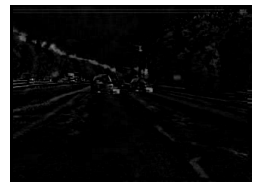

(c)

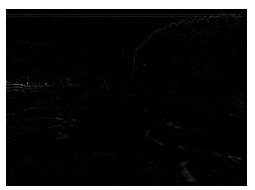

(d)

Fig. 2. Stabilization measure on sequence $C A R$ (a) $3^{\text {rd }}$ frame; Difference of $3^{\text {rd }}$ and $4^{\text {th }}$ frame (b) Original (c) CW (d) Proposed

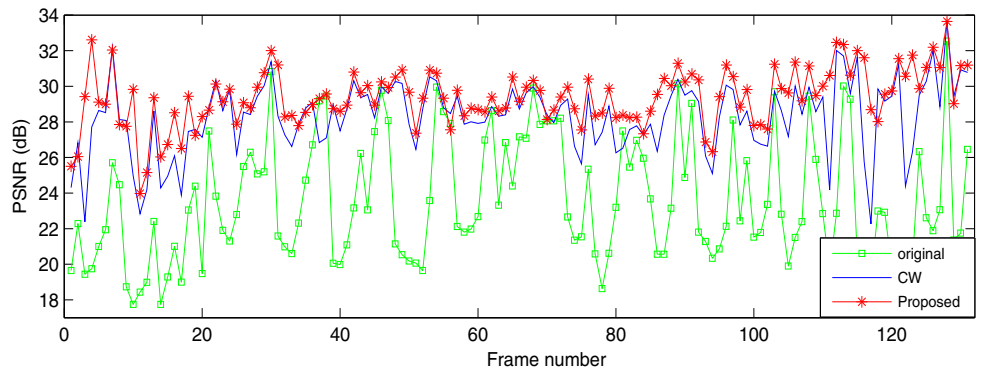

Fig. 3. Comparison of PSNR values for complete video $C A R$

intensity based stabilization techniques get affected by such variations and results in wrong motion estimation. The proposed DCW technique provides better stabilization due to accurate alignment of projection curves. In terms of motion accuracy, the CW [5] has earlier been suggested as the most efficient over the existing techniques. Efficiency of the proposed method over CW method for moving platform applications is evaluated using difference frame analysis, shown in Fig. 2. Fig. 2(a)-(d) shows the $3^{\text {rd }}$ frame of the video $C A R$, original difference frame of $3^{r d}$ and $4^{\text {th }}$ frame, corresponding stabilized results using $\mathrm{CW}$ and proposed DCW method respectively. The darker difference frame of DCW in comparison to $\mathrm{CW}$ method signifies better frame alignment. The proposed method is proved to be robust over CW in terms of accurate matching of projection curves. Comparison of the two methods is also done using estimated PSNR values corresponding to each pair of consecutive frame [3]. Proposed technique gives better PSNR as shown in Fig. 3. To validate the efficiency and robustness of the proposed method, various real-world moving platform videos, each of resolution $240 \times 320$ are chosen for performance analysis purpose. Fig. 4 shows the different test videos taken from moving bus. The performance evaluation is done in terms of interframe transformation fidelity (ITF) and processing time. ITF is an averaged PSNR value calculated between each consecutive pair of frames over entire video. Stabilized sequence results in a higher ITF value. Comparison of various stabilization techniques for different test videos is given in Table 1. Proposed technique proves to be less time efficient in comparison to GCBPM and IP methods but gives better stabilization over existing methods. The robustness of the proposed algorithm is achieved in terms of accurate motion estimation. 


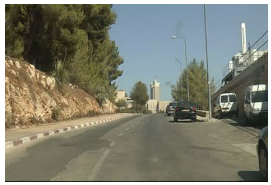

(a)

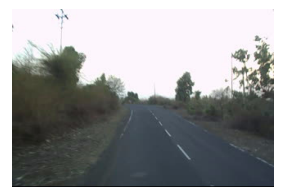

(b)

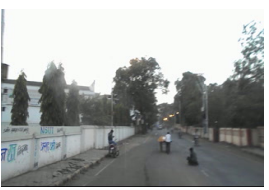

(c)

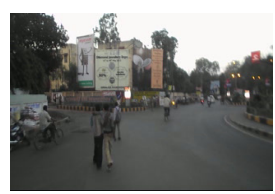

(d)

Fig. 4. Other moving platform test video (a)Test1 (b)Test2 (c)Test3 (d) Test4

Table 1. Comparative results of the ITF and processing speed

\begin{tabular}{|c|c|c|c|c|c|c|c|c|c|c|c|}
\hline \multirow{3}{*}{$\begin{array}{c}\text { Test } \\
\text { Video }\end{array}$} & \multicolumn{6}{|c|}{ ITF } & \multirow{2}{*}{\multicolumn{5}{|c|}{$\begin{array}{l}\text { Processing time } \\
\text { (sec/frame) }\end{array}$}} \\
\hline & \multirow[t]{2}{*}{ Orig. } & \multicolumn{5}{|c|}{ Stabilized } & & & & & \\
\hline & & $\overline{\mathrm{BM}}$ & GCBPM & IP & CW & Proposed & $\overline{\mathrm{BM}}$ & GCBPM & IP & CW & Proposed \\
\hline Test1 & 21.71 & 21.94 & 22.65 & 22.89 & 23.16 & 24.33 & 2.124 & 0.107 & 0.059 & 40.72 & \begin{tabular}{|l}
0.148 \\
\end{tabular} \\
\hline Test2 & 20.10 & 21.35 & 23.32 & 24.10 & 24.73 & 26.89 & 2.190 & 0.102 & 0.070 & 40.11 & 0.137 \\
\hline Test3 & 20.44 & 20.83 & 21.69 & 23.57 & 24.03 & 24.74 & 2.018 & 0.092 & 0.069 & 38.96 & 0.128 \\
\hline Test4 & 22.48 & 23.71 & 24.01 & 25.63 & 25.97 & 27.64 & 2.316 & 0.130 & 0.063 & 40.61 & 0.156 \\
\hline
\end{tabular}

\section{Conclusion}

This paper introduces a new derivative dynamic time warping based video stabilization technique. Robustness of the proposed technique has been discussed in the field of moving platform videos. The proposed DCW method provides better stabilization in terms of accurate projection curve alignment, providing higher ITF value in comparison to the existing intensity based stabilization techniques. Future work will focus on improving the time efficiency of the proposed algorithm to make it useful for real-time application.

\section{References}

1. Vella, F., Castorina, A., Mancuso, M., Messina, G.: Digital image stabilization by adaptive block motion vectors filtering. IEEE Transactions on Consumer Electronics 48(3), 796-801 (2002)

2. Ko, S.J., Lee, S.H., Jeon, S.W., Kang, E.S.: Fast digital image stabilizer based on gray-coded bit-plane matching. IEEE Transactions on Consumer Electronics 45(3), 598-603 (1999)

3. Li, S., Qi, J.: Image stabilization by combining gray-scale projection and representative point matching algorithms. In: 3rd International Conference on Awareness Science and Technology (iCAST 2011), pp. 128-132 (September 2011)

4. Piva, S., Zara, M., Gera, G., Regazzoni, C.S.: Color-based video stabilization for realtime on-board object detection on high-speed trains. In: IEEE Conference on Advantage Video and Signal Based Surveillance (AVSS 2003), pp. 299-304 (July 2003)

5. Bosco, A., Bruna, A., Battiato, S., Bella, G., Puglisi, G.: Digital video stabilization through curve warping techniques. IEEE Transactions on Consumer Electronics 54(2), 220-224 (2008)

6. Keogh, E.J., Pazzani, M.J.: Derivative dynamic time warping. In: The First SIAM International Conference on Data Mining (SDM 2001), pp. 1-11 (April 2001) 